\title{
ALTERATIONS IN THE STRUCTURE, FUNCTION, AND CHEMISTRY OF C FIBERS FOLLOWING LOCAL APPLICATION OF VINBLASTINE TO THE SCIATIC NERVE OF THE RAT ${ }^{1}$
}

\author{
MARIA FITZGERALD, ${ }^{2}$ CLIFFORD J. WOOLF, SALLY J. GIBSON, ${ }^{*}$ AND PETER S. MALLABURN \\ Department of Anatomy, University College London, Gower Street, London WC1E 6BT and *Histochemistry Unit, Department of \\ Histopathology, Royal Postgraduate Medical School, Hammersmith Hospital, London W12 OHS, United Kingdom
}

Received May 16, 1983; Revised August 23, 1983; Accepted August 23, 1983

\begin{abstract}
Vinblastine, a transport blocker, was applied locally to the sciatic nerve in rats. It was found to be a powerful neurotoxin with a dose-dependent action, destroying all afferents at doses of $5 \times 10^{-4}$ $\mathrm{M}$, primarily C fibers at intermediate doses of $2.5 \times 10^{-4} \mathrm{M}$, and only at a critically low dose of $10^{-4}$ $M$ was a degeneration-free axon transport blockade, lasting for 4 to 5 days, produced. Such transport block failed to alter thermal responsiveness of the rats as measured behaviorally, by the flexor reflex, or by dorsal horn cell responses. It did, however, significantly reduce both the chemical sensitivity of the $\mathrm{C}$ afferents and their ability to produce neurogenic edema. This began $24 \mathrm{hr}$ after treatment and lasted 4 to 5 days. Therefore, it is likely that these functions are dependent on the continuous transport of some compound to the axon terminals from the cell body. This low concentration of local vinblastine treatment also resulted in depletion of fluoride-resistant acid phosphatase from $\mathrm{C}$ fiber terminals in the dorsal horn of the spinal cord. Transmission from $\mathrm{C}$ fibers to second-order neurons in the spinal cord, however, was totally unaffected. Substance P levels in the spinal terminals was largely unaffected, although in 1 of 5 cases there was depletion. It appears, therefore, that some, but not all, retrograde changes in sensory neurons following peripheral nerve damage can be mimicked by blockade of axon transport. The effects following vinblastine treatment are compared to other peripheral nerve manipulations, such as cut, crush, and application of local capsaicin.
\end{abstract}

It is well established that a variety of different materials are actively transported from their site of production in the cell body of a neuron to their site of action in the dendrites, axons, or axonal terminals. Conversely, there is clear evidence for retrograde transport mechanisms returning material from the periphery to the cell body for degradation or restoration (see Schwartz, 1979). However, in addition to this role for active transport in maintaining their structural and functional integrity, neurons also transport chemicals which are essential for the integrity of second-order structures which they innervate. An example of such a "trophic" function is seen on taste buds which degenerate if axonal transport in the lingual nerve is blocked (Sloan et al., 1983). Cutaneous nerves are known to release material from their peripheral terminals in response to activation via an

\footnotetext{
${ }^{1}$ This work was supported by the Medical Research Council and the Wellcome Trust. We are greatly indebted to C. King, A. Ainsworth, P. Ainsworth, and J. Bannon for their invaluable assistance. We also gratefully thank Professor P. D. Wall for his many useful suggestions.

${ }^{2}$ To whom correspondence should be addressed.
}

axon reflex (Lewis, 1942; Holton, 1959; Chapman et al., 1961; Jancso, 1968). It is possible that this, too, is an example of such a trophic function.

Another role proposed for retrograde transport is to carry signals to inform the cell body about events in the periphery (Kristensson and Olsen, 1974; Stoekel et al., 1975; Bisby and Bulger, 1977). When a peripheral nerve is cut, apart from the Wallerian degeneration of the distal axon and local changes at the site of the lesion, there are extensive changes far proximal to the cut which take from days to weeks to occur (Lieberman, 1971). Examples are chromatolysis of dorsal root ganglion cells and changes in the chemical content and structure of primary afferent terminals in the dorsal horn (Csillik and Knyihar, 1978; Barbut et al., 1981). There is evidence, moreover, that this signal may also alter the function of second-order cells in the dorsal horn, again suggesting a trophic function of nerves over their innervated structures, but this time in the CNS (see Wall, 1983). Clearly, then, it is possible that axonal transport in primary afferent sensory neurons may have several different functions. One may be to maintain innervated structures, 
either centrally or peripherally, and another may be to signal any changes in the environment of their distant processes.

In the following series of experiments we have looked for evidence for these two proposed functions in primary afferent $\mathrm{C}$ fibers following the blockade of their active transport with the microtubule inhibitor vinblastine. To do this we have looked at the behavioral, electrophysiological, anatomical, and chemical consequences of a brief local application of vinblastine to the sciatic nerve on peripheral cutaneous sensory receptors and on the central C-terminals in the dorsal horn.

\section{Materials and Methods}

The experiments were performed on Wistar rats of both sexes weighing between 200 and $300 \mathrm{gm}$.

\section{Vinblastine treatment}

Rats were anesthetized with pentobarbitone, $(40 \mathrm{mg} /$ $\mathrm{kg}$, i.p.). Using sterile procedures, the sciatic nerve in the left leg was exposed in the popliteal fossa, dissected free, and placed on a piece of Parafilm to separate it from the surrounding muscle. Small pledgets of cotton wool soaked in $10^{-5} \mathrm{M}, 10^{-4} \mathrm{M}, 2.5 \times 10^{-4} \mathrm{M}, 5 \times 10^{-4} \mathrm{M}$, or $10^{-3} \mathrm{M}$ vinblastine in $0.9 \%$ saline were then gently packed around a $10-\mathrm{mm}$ length of nerve and left in place for 15 min. They were then removed along with excess fluid, and the wound was closed in two layers. The animals all recovered uneventfully.

\section{Axon transport blockade}

At various times following different doses of vinblastine treatment, rats were anesthetized with pentobarbitone $(40 \mathrm{mg} / \mathrm{kg}$, i.p. $)$ and the sciatic nerve was ligated distal to the site of vinblastine application, under sterile conditions. The wound was closed and the animals were allowed to survive a further $24 \mathrm{hr}$. Following that, they were perfused with $10 \%$ formalin, and the sciatic nerves were removed. These nerves were cut in longitudinal sections and stained for fluoride-resistant acid phosphatase (FRAP) (see "Chemistry of afferent terminals in the dorsal horn") or acetylcholinesterase (AChE) (Kiernan, 1981). The accumulation of the enzymes at the ligature indicated intact axonal transport, whereas their absence indicated transport blockade. The method used for measurement was therefore qualitative, not quantitative.

\section{Morphology}

At various times following vinblastine treatment, the morphology of the nerves was investigated.

Electron microscopy of the sciatic nerve. Treated and untreated animals were perfused with half-strength Karnowski's fixative, and the sciatic nerves were removed and prefixed in the same for $2 \mathrm{hr}$. After osmium postfixation, the tissue was taken through an ethanol and propylene oxide row and embedded in Araldite. Ultrathin longitudinal or transverse sections were mounted on naked copper grids, stained with uranyl acetate and lead citrate, and viewed in a Philips 300 electron microscope to determine whether vinblastine treatment had produced any morphological changes in A or C fibers.

Light microscopy. The rats were perfused with $10 \%$ formaldehyde and the sciatic nerves were removed and stained using the Marchi method for degenerating myelin (Kiernan, 1981). Nerves were then teased out into individual fibers on a glass slide and inspected under the light microscope. Degenerating myelin stains black and is fragmented, appearing as rows of black dots.

\section{Behavioral testing}

Behavioral tests to determine the integrity of the sensory innervation of the foot were performed at various times after the vinblastine treatment. The tactile placing reflex was tested by touching the lateral dorsal surface of the foot of a suspended animal on the edge of a bench top to see if low threshold afferent input was intact. Sensitivity to stronger mechanical stimuli was tested by pinching the lateral foot and toe with a pair of calibrated forceps producing $150 \mathrm{gm} / \mathrm{mm}^{2}$. The response to noxious thermal stimuli was tested by dipping the lateral side foot into a thermostatically controlled hot water bath, maintained at $48^{\circ} \mathrm{C}$. The latency of response was measured as the time between immersion of the foot in hot water to the time of flexor withdrawal from it. This withdrawal was always brisk and clearly defined. Each foot was tested three times per trial, alternating between left and right foot. The latencies were very repeatable from trial to trial in a given rat, although there was some interanimal variation. If there was any sign of a motor paralysis with abnormal gait or foot drop, the animal was immediately sacrificed.

\section{Neurophysiology}

Neurophysiological investigations were made of changes in cutaneous receptor function, transmission, and spinal cord function following vinblastine treatment. Two types of anesthesia were used: $25 \%$ urethane intraperitoneally (for experiments a and c, see below), and Althesin intravenously after initial induction with ether (experiment b). The trachea and carotid artery were cannulated in all animals, and rectal temperature, blood pressure, heart rate, and expired $\mathrm{CO}_{2}$ were continuously monitored and maintained within a normal physiological range.

(a) Conduction along the sciatic nerve. The treated and untreated sciatic nerves in both legs were exposed in the popliteal fossa from the sciatic notch to the sural nerve and covered with warm mineral oil. The temperature of the oil pool was maintained at 34 to $36^{\circ} \mathrm{C}$ by overhead lamps. The sciatic nerves were then placed on a pair of silver stimulating electrodes 10 to $30 \mathrm{~mm}$ proximal to a pair of silver recording electrodes positioned on the sural nerve which was cut distally. Stimulus threshold, conduction latency, and compound action potential amplitude at 2 times threshold were measured for both the A and $\mathrm{C}$ compound action potentials traveling across the treated length of nerve or its equivalent on the control side. To evoke the A waves, stimulation at 10 to $500 \mu \mathrm{A}$, $50 \mu \mathrm{sec}$ at $1 \mathrm{~Hz}$ was used; for $\mathrm{C}$ waves the stimulation was 1 to $10 \mathrm{~mA}, 500-\mu \mathrm{sec}$ pulses at $0.5 \mathrm{~Hz}$. Conduction velocity was measured with a single stimulus and recording site using the latency between the start of the stimulus artifact and the beginning of the rising phase of the compound action potential. 
(b) Flexor EMG recordings. These tests were an indirect way of looking at both cutaneous receptor and dorsal horn function following treatment. Needle electrodes were placed in the hamstring muscles in the back of the leg, and a multi-unit EMG response to natural stimulation of the lateral hindfoot and toe was recorded. Before making any recordings the saphenous nerve was cut at the level of the knee, so that the foot was only innervated by the sciatic nerve. The skin was pinched with a pair of standardized forceps applying $150 \mathrm{gm} / \mathrm{mm}^{2}$, heated to $50^{\circ} \mathrm{C}$ with a radiant heat lamp, and then the irritant chemical, mustard oil, was applied to the skin surface. Both treated and untreated sides were studied.

(c) Dorsal horn cell recordings. These were made to see whether vinblastine treatment altered the receptive field properties of dorsal horn neurons. A laminectomy was performed to expose the lumbar enlargement of the spinal cord, which was covered with warm mineral oil. The sciatic nerves on both sides were exposed and placed on silver wire stimulating electrodes proximal to the site of treatment. The saphenous nerves on both sides were cut. Recordings of dorsal horn cell activity were made with KCl-filled glass microelectrodes of 5 to 7 megohms impedance. Repeated parallel tracks in the L4 segment, the region of termination of the sciatic nerve, were made on both sides, recording all clear unitary potentials in each track between 400 and $1000 \mu \mathrm{m}$ from the surface. Receptive fields were characterized in terms of their response to mechanical stimuli, using brushing, touching, pinching with forceps, and to radiant heating $\left(50^{\circ} \mathrm{C}\right)$. In addition, for each unit, the responses evoked by electrical stimulation of the $\mathrm{A}$ and $\mathrm{C}$ fibers in the sciatic nerve were recorded.

\section{Neurogenic edema}

Rats were anesthetized with $25 \%$ urethane and their carotid artery was cannulated. Evans blue $(1 \%, 5 \mathrm{ml} / \mathrm{kg})$ was then injected into the bloodstream. Neurogenic edema, which is a $\mathrm{C}$ fiber-mediated peripheral action, was evoked either by the application of $50 \%$ mustard oil to the lateral foot or by stimulation of the sciatic nerve at $5 \mathrm{~Hz}$ at a strength sufficient to excite $\mathrm{C}$ fibers $(5 \mathrm{~mA}$, $500 \mu \mathrm{sec})$. The presence of neurogenic extravasation was seen by the appearance of a blue patch on the skin, and this was quantified by extraction of the Evans blue dye from a piece of the relevant skin with formamide. Using spectrophotometry, it was then possible to calculate the amount of dye extravasated per unit weight of skin (Jancso et al., 1967).

\section{Chemistry of afferent terminals in the dorsal horn}

At various times after vinblastine treatment, the levels of the chemicals FRAP and substance P (SP), known to be present in $\mathrm{C}$ fiber afferents in the dorsal horn, were investigated.

$F R A P$. The rats were perfused with calcium/formalin and the spinal cord was removed after identification of the segments by dissection of the sciatic nerve. Transverse sections were cut through the lumbar cord and stained for FRAP as described previously (Ainsworth et al., 1981).
$S P$. The rats were perfused with parabenzoquinone $(0.4 \% \mathrm{w} / \mathrm{v})$ in phosphate-buffered saline and the cord was removed. Transverse sections were stained for SP by the peroxidase-antiperoxidase (PAP) method described in detail elsewhere (Ainsworth et al., 1981).

FRAP or peptide staining was assessed by eye in at least 20 sections per animal, comparing the density of stain on the side of the treated sciatic nerve and on the untreated side and with animals where the sciatic nerve had been cut or crushed or left intact. In one animal stained for FRAP, serial sections were drawn on the camera lucida and the total area of depletion along the cord was reconstructed.

\section{Results}

The first series of experiments was performed using $10^{-3} \mathrm{M}$ vinblastine, since this is a dose that has been used in previous studies (Cull, 1975; Csillik et al., 1978). However, it soon became clear that treatment with this concentration always resulted in motor paralysis and anesthesia due to nerve degeneration; therefore, the dose was reduced to $5 \times 10^{-4} \mathrm{M}$. But, as will be seen in the results below, this dose and $2.5 \times 10^{-4} \mathrm{M}$ still produced some nerve damage which varied between different animals. We ultimately found that a dose of $10^{-4} \mathrm{M}$ was the most satisfactory, producing transport block without degeneration, whereas $10^{-5} \mathrm{M}$ was ineffective in producing transport block.

\section{Effects of vinblastine on the axons}

Axonal tranport. Axon transport of both AChE and FRAP was effectively blocked by doses down to $10^{-4} \mathrm{M}$ $(n=10)$. A dose of $10^{-5} \mathrm{M}$ vinblastine failed to affect axon transport $(n=2)$. The earliest time tested for $10^{-4}$ $M$ vinblastine was 36 to $48 \mathrm{hr}$ after treatment and, at this time, accumulation of chemicals proximal to a ligation was totally absent on the treated side (Fig. 1). It remained absent until day 4 to 5 . Distal accumulation was normal at $48 \mathrm{hr}$ but not after that time. Transport in the opposite untreated nerve was always intact (Fig. 1). By day 5 to 6 following $10^{-4} \mathrm{M}$ treatment, axonal transport was beginning to recover, and by day 10 to 11 accumulation was indistinguishable from control (Fig. 1).

Nerve conduction. The highest dose used, $5 \times 10^{-4} \mathrm{M}$, had a severe effect on conduction of both the $A$ wave and the $\mathrm{C}$ wave (see Fig. 2). One day after treatment the $\mathrm{A}$ wave was completely normal $(n=4)$, but by day 2 it was beginning to fall in size and become slower $(n=3)$. On day 3 , it was smaller still and collapsed after several repeated stimuli. In three of four cases on day 4 , no $A$ wave was present at all, and in the other case it was very small (Fig. 2, $A$ and $B$ ). The $\mathrm{C}$ wave was already reduced in amplitude on day 1 , and from day 2 onward was either not present or very small and unable to follow more than one stimulus (Fig. $2 C$ ). The lowest dose used, $10^{-5} \mathrm{M}$, did not affect conduction in the $\mathrm{A}$ or the $\mathrm{C}$ fibers, as tested on day $3(n=4)$. Between these doses it was clear that a differential effect occurred. The A wave was completely normal following 2.5 or $1.0 \times 10^{-4} \mathrm{M}$ treatment (Fig. 2, $A$ and $B$ ), but the $C$ wave was severely affected (Fig. $2 C$ ). 

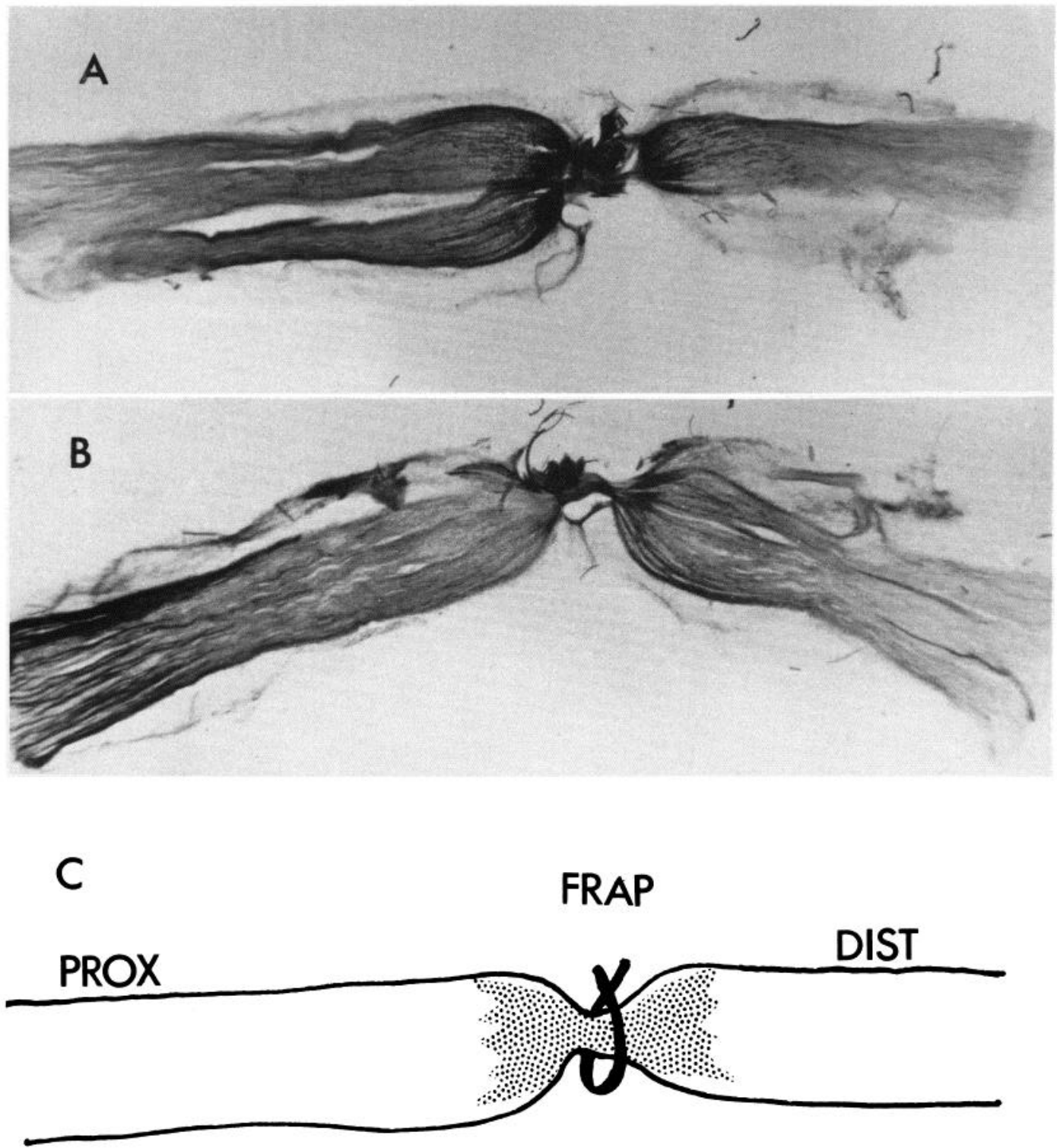

Figure 1. The acummulation of FRAP shown in longitudinal sections of the sciatic nerve proximal and distal to a ligature made $24 \mathrm{hr}$ before. $A$, A control untreated nerve. $B$, A nerve that was treated with $10^{-4} \mathrm{M}$ vinblastine at a proximal site $24 \mathrm{hr}$ before the ligature. No accumulation of FRAP occurs proximal to the tie, although some can be seen distal to it. Note the FRAP accumulation proximal to the site of vinblastine application. $C$, A schematic diagram of the nerve sections.

Three days after a $10^{-4} \mathrm{M}$ dose, a $\mathrm{C}$ wave could not be recorded in a third of the treated nerves $(n=12)$, and in the remaining two-thirds the $\mathrm{C}$ wave was very small. Nerves on the opposite side were unaffected. By day 7 , the $\mathrm{C}$ wave, although small, was always present $(n=4)$ and by day 14 was definitely recovered $(n=3)$ (Fig. $2 C$ ). Interestingly, the conduction velocity of the $C$ wave was not affected by the treatment, only the amplitude.

EM studies of the nerve. Myelin staining with the Marchi stain, together with teasing of the nerve and inspection under the light microscope, revealed that $5 \times$ $10^{-4} \mathrm{M}$ vinblastine treatment always resulted in considerable degeneration in the myelinated fibers of the nerve $(n=6)$. This was seen as a fragmenting of the myelin by the second day after treatment, which agrees well with the failure of conduction at this time (see "Nerve conduction"). EM studies were necessary for lower doses and to study effects on unmyelinated fibers. Figure 3, $a$ to $c$, demonstrates typical EM sections of nerve distal to the site of $2.5 \times 10^{-4} \mathrm{M}$ vinblastine treatment 4 days earlier. Four nerves were examined under the electron microscope. Damage was found to be predominantly in the unmyelinated fibers. Scanning over sections at low power revealed, first, that the $\mathrm{C}$ fibers were considerably reduced in number. Close inspection showed that many of the $\mathrm{C}$ fibers that remained showed evidence of degeneration. The axons were often filled with granular debris, neurofilaments were disrupted, and some axons were 

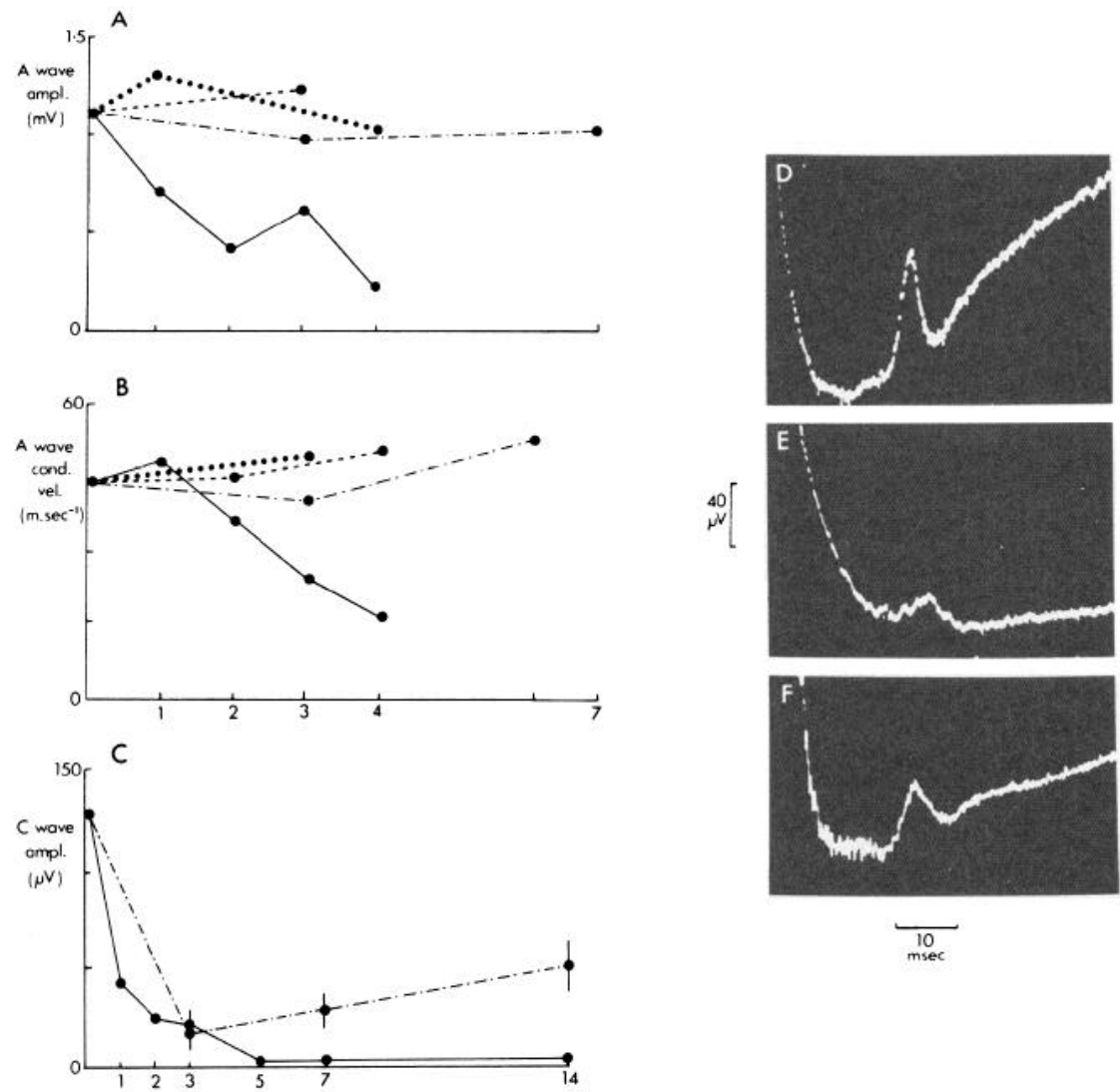

Figure 2. Conduction along the sciatic nerve through the site of vinblastine application. Doses are represented as: $5 \times 10^{-4} \mathrm{M},-; 2.5 \times 10^{-4} \mathrm{M}$,

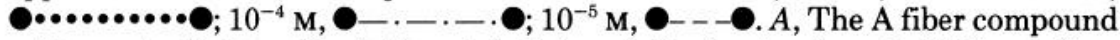
action potential amplitude from 1 to 7 days following treatment. $B$, The A fiber compound action potential conduction velocity 1 to 7 days following treatment. $C$, the $\mathrm{C}$ fiber compound action potential amplitude from 1 to 14 days following treatment. $D, E$, and $F$ are representative records of $\mathrm{C}$ waves evoked from sciatic nerves stimulated at $5 \mathrm{~mA}, 500 \mu$ sec: untreated $(D)$, and treated with $10^{-4} \mathrm{M}$ vinblastine for 3 days $(E)$ and 14 days $(F)$ previously. Conduction distance was $25 \mathrm{~mm}$.

greatly enlarged and contained an aggregation of organelles (Fig. $3 c$ ). In some cases (Fig. $3 b$ ) the $\mathrm{C}$ fiber axolemma was completely fragmented, leaving freely lying collections of debris and organelles in the endoneural space. The changes in the $\mathrm{C}$ fiber axon morphology were often accompanied by changes in their Schwann cells including the presence of flattened processes identical to that described by Thomas and King (1974). Many macrophages were present engulfing the axonal debris. In some nerves myelinated axons were also clearly degenerating, showing characteristic darkening of the axoplasm, retraction of the axolemma, and a separation and fragmentation of the myelin (Fig. $3 b$ ). Increased mitotic activity occasionally was observed in the Schwann cells. The extent of myelinated axon damage was variable, being nonexistent in one case, minimal in another, and definitely present although not extensive in the remaining two cases. The $\mathrm{C}$ fiber damage in contrast was considerable in all of the nerves at this dose.

At the lowest dose, $10^{-4} \mathrm{M}$, no A or $\mathrm{C}$ fiber degeneration was observed on electron microscopic examination of two nerves tested at 4 days and 9 days following treatment. Figure $3 d$ shows a typical bundle of $\mathrm{C}$ fibers following $10^{-4} \mathrm{M}$ vinblastine. This indicates that, at this dose, effective axon transport blockade can be achieved for 4 to 5 days (see "Axonal transport") in the absence of clear morphological signs of degeneration.

\section{Effects of vinblastine on primary afferent terminals in the dorsal horn}

FRAP. FRAP was depleted from the central terminals of $\mathrm{C}$ fibers in lamina 2 of the dorsal horn by treatment of the sciatic nerve with $10^{-4} \mathrm{M}$, or 2.5 or $5.0 \times 10^{-4} \mathrm{M}$ vinblastine $(n=10)$ (Fig. 4). The depletion was first detectable on day 4 to 5 post-treatment and was still present on day 4. A map of the extent of the depletion was drawn from the spinal cord of an animal treated with $10^{-4} \mathrm{M} 10$ days previously. The gap stretched from L3 to L5 and appeared identical to that following nerve section described by Devor and Claman (1980).

$S P$. The effect of vinblastine treatment of the sciatic nerve on SP distribution in the dorsal horn was tested 

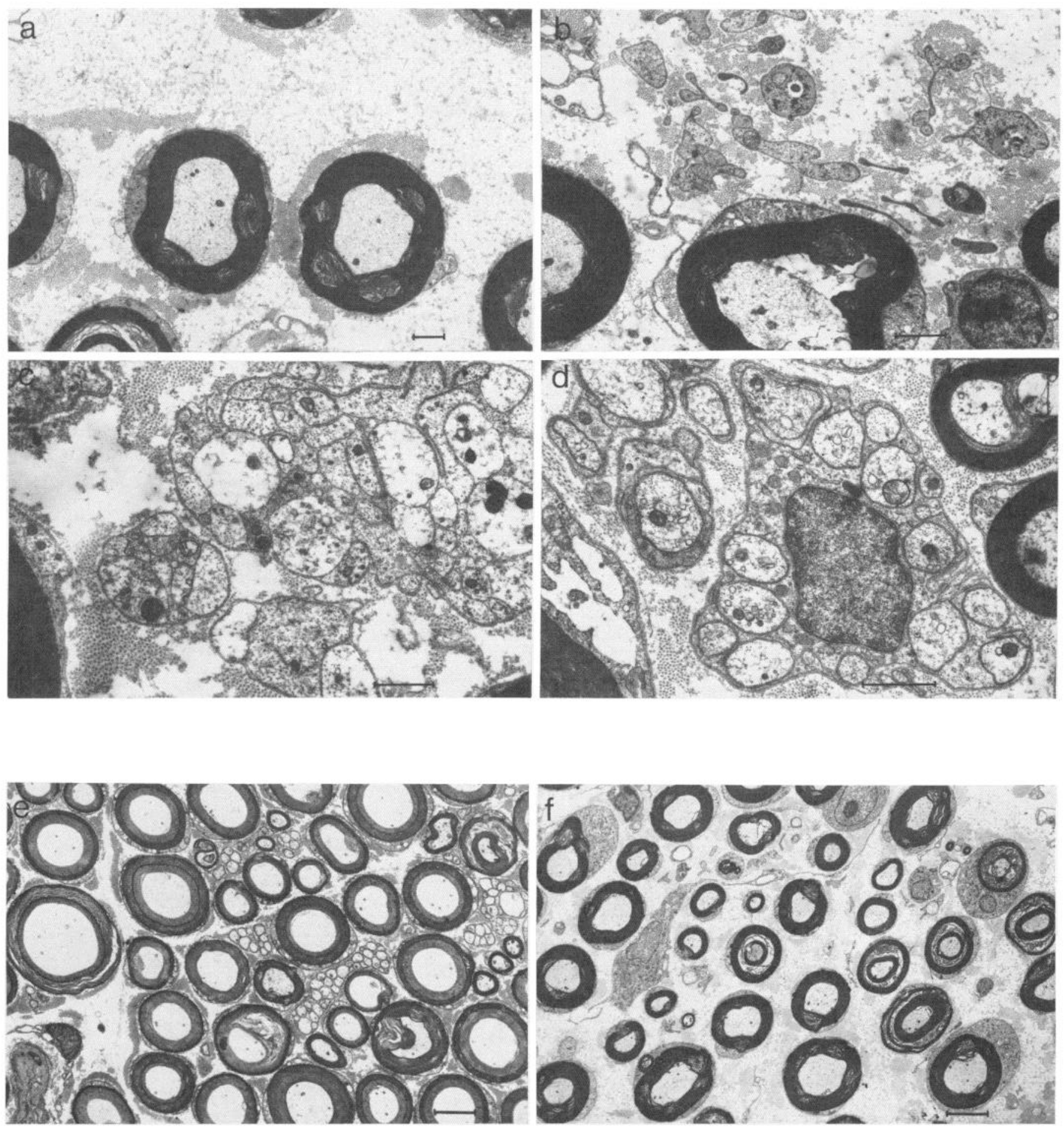

Figure 3. Electron micrographs of sciatic nerves following their treatment with vinblastine 4 days previously. In each case sections were made from the nerve just distal to the site of application. $a, b$, and $c$ show in high power the changes in the nerve produced by $2.5 \times 10^{-4} \mathrm{M}$ vinblastine. In $a$ there is increased endoneurial fluid accumulation with no apparent $\mathrm{C}$ fibers. In $b$ there are typical flattened processes of Schwann cells found in Wallerian degeneration associated here with a degenerating $\mathrm{A}$ fiber. In $c$ is a micrograph of degenerating swollen $\mathrm{C}$ fibers with accumulation of organelles and disrupted neurolemmal membranes. In $d$ the nerve has been treated with a lower dose $\left(10^{-4} \mathbf{M}\right)$ of vinblastine, and this illustrates a Schwann cell containing normal $\mathrm{C}$ fibers. $e$ and $f$ are lower power micrographs to illustrate the density of $\mathrm{C}$ fibers in a normal untreated sciatic nerve $(e)$ and a nerve pretreated 4 days earlier with $2.5 \times 10^{-4} \mathrm{M}$ vinblastine $(f)$. The scale bars in $a$ and $b$ are $2 \mu \mathrm{m}$, those in $c$ and $d$ are $1 \mu \mathrm{m}$, and those in $e$ and $f$ are $5 \mu \mathrm{m}$. 
only with the high dose of $5 \times 10^{-4} \mathrm{M}$ vinblastine. The results proved to be variable. In four animals, tested 6 , 12,14 , and 18 days after treatment, the levels of substance $\mathrm{P}$ as judged by the density of the PAP reaction in

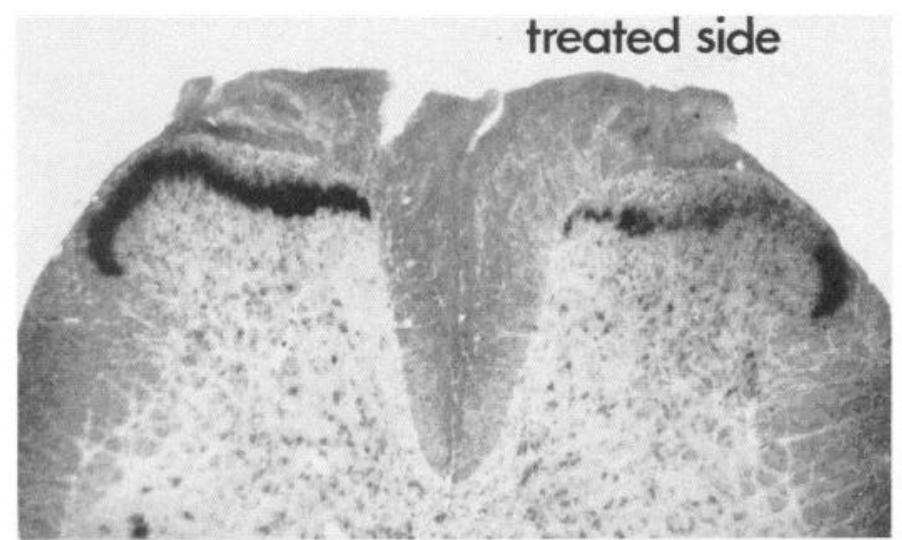

Figure 4. Cross-section of the spinal cord at L4 segment stained for FRAP. The sciatic nerve on one side (right) was treated with $10^{-4} \mathrm{M}$ vinblastine 10 days before. FRAP is located in a thin band of afferent $\mathrm{C}$ fiber terminals in the superficial dorsal horn. On the treated side, however, FRAP is depleted in the region of termination of the sciatic nerve. the dorsal horn were totally unaffected, but in one other animal (day 14) there was clear depletion of SP in the area of termination of the sciatic nerve.

Dorsal horn cell responses. The responses to natural skin stimulation of the foot and to electrical activation of primary afferents in the treated sciatic nerve of dorsal horn cells were tested in four animals at $10^{-4} \mathrm{M}$ (two at day 4 and two at day 7). Twenty cells were recorded on both sides of the cord in each animal. In all cases the results were not significantly different from control. Stimulation of the sciatic nerve proximal to the site of treatment revealed that all cells had an input from A fibers, and 50 to $60 \%$ cells responded with a long latency burst to $\mathrm{C}$ fiber stimulation at 100 to $120 \mathrm{msec}$. Spontaneous activity was normal, and responses to brushing, pressure, pinching, and heating the skin of the foot were no different from those found in untreated animals (Fitzgerald, 1982; Fitzgerald and Woolf, 1982). The proportions of wide dynamic range, low threshold, and noxious specific cells were also unchanged.

\section{Effects on reflexes and neurogenic edema}

Behavioral reflexes. In view of the severe conduction failure and degeneration found following treatment of the sciatic nerve with a high dose of $5 \times 10^{-4} \mathrm{M}$ vinblas-

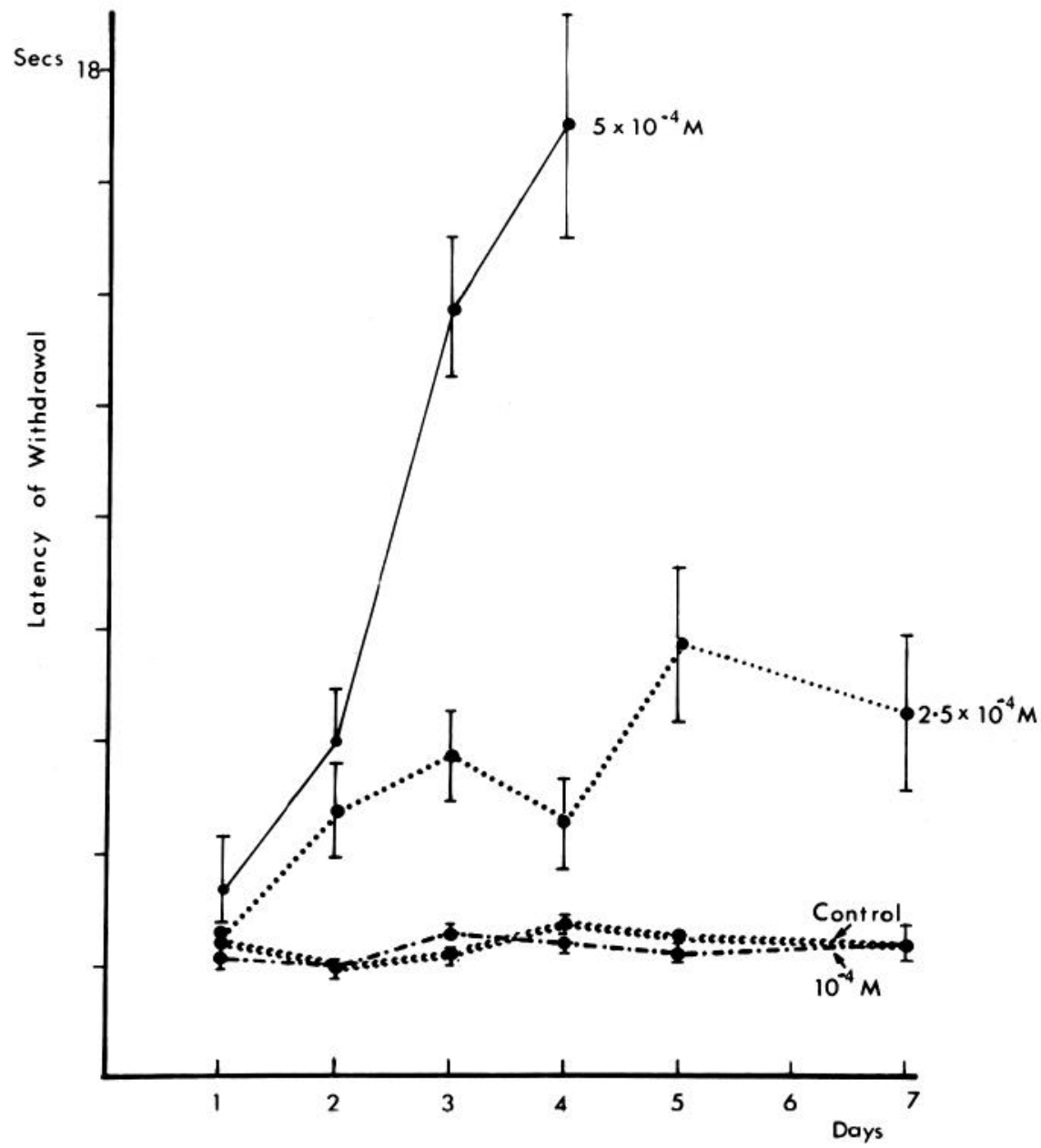

Figure 5. Latencies of foot withdrawal from water at $48^{\circ} \mathrm{C}$. The sciatic nerve was treated with various doses of vinblastine 1 to 7 days before. 

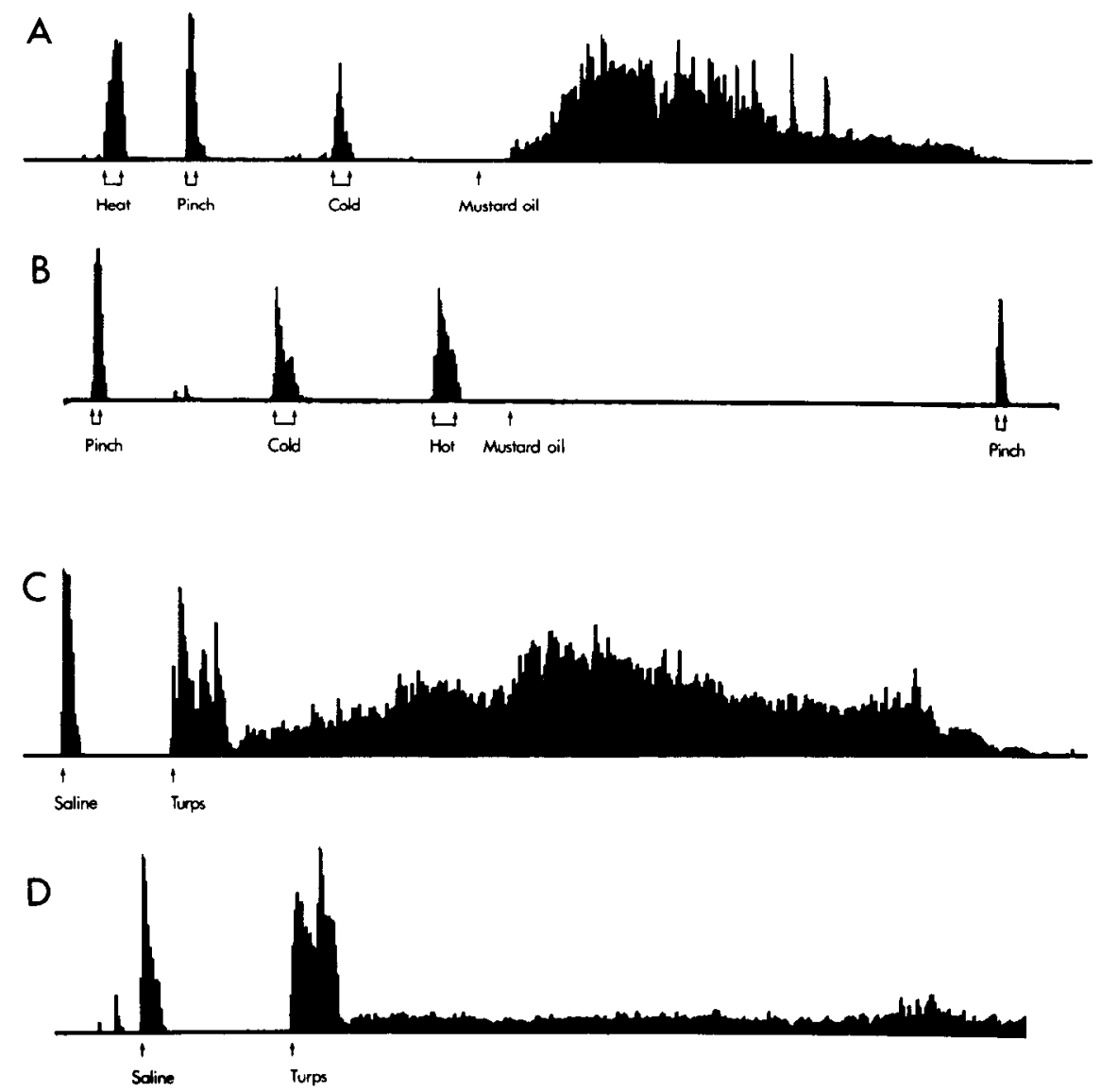

Figure 6. Rate meter records of multi-unit EMG responses recorded from the hamstring muscles. The LHS sciatic nerve was treated with $10^{-4} \mathrm{M}$ vinblastine 3 days earlier. $A$, Flexor reflexes are evoked from heating, pinching, cold, and mustard oil on the untreated side. $B$, On the treated side, flexor reflexes can be evoked from heat, pinch, and cold but not from mustard oil. $C$, Turps injected into the foot on the untreated side evoked a long-lasting response, but on the treated side $(D)$ turps evoke an early burst only.

tine, it is not surprising that these animals also developed a marked insensitivity to both mechanical pinching and noxious thermal stimulation of the treated hindlimb $(n$ $=8$ ). Figure 5 shows the increase in latency to foot withdrawal which becomes apparent on the second day after treatment. The extent of analgesia was quite variable from animal to animal. Tactile placing reflexes and motor control appeared totally unaffected. At a lower dose of $2.5 \times 10^{-4} \mathrm{M}(n=22)$ the effects were less profound, but latency of withdrawal to hot water was still significantly increased, and some deficit in responses to pinch was also observed. Again, the effects were variable. A small proportion of animals ( $10 \%)$ were totally unaffected. At $10^{-4} \mathrm{M}$, a dose which produced no degeneration of the nerve ("EM studies of the nerve"), there was absolutely no effect in any animals on the reflex withdrawal behavior either to pinching or to heating ( $n$ $=24$ ), from the first day after treatment until 14 days later (Fig. 5).

Flexor EMG responses. EMG responses from the hamstring muscles evoked by noxious heating of the hindfoot confirmed the above results. Four animals were tested on each day from day 1 to 5 and then day 7 and day 14 following $10^{-4} \mathrm{M}$ vinblastine treatment. At no stage did the flexor EMG response to either pinching the skin, heating it to $50^{\circ} \mathrm{C}$, or cooling it to $0^{\circ} \mathrm{C}$ differ from the opposite, untreated side. In contrast to this, there was a clear deficit in the flexor response to irritant chemicals applied to the foot. Both the duration and the amplitude of the characteristic high frequency outburst to mustard oil painted on the skin of the lateral foot were significantly reduced on the treated side. Figure $6, A$ and $B$, shows some typical EMG rate meter records of this. Peak amplitudes and durations of EMG responses were measured on both sides. The results are shown in Figure 7, $A$ and $B$. The deficit was not apparent until the second day after treatment, the responses on day 1 being essentially normal. This block lasted until day 4 to 5 and recovered on day 7. Injection of turpentine, subcutaneously, also produced a much decreased and curtailed response compared to controls. In seven animals tested on days 2 and 3 and two tested on day 4 the early burst of 10 to $15 \mathrm{sec}$ on injection was normal, but the later, longer-lasting response was reduced in amplitude and duration. The intensity of effect was variable, but the mean reduction of duration on the treated (LHS) side as compared to the untreated (RHS) side was $39 \%$ and the mean reduction of amplitude was $48 \%$. Figure $6, C$ and $D$, shows an 

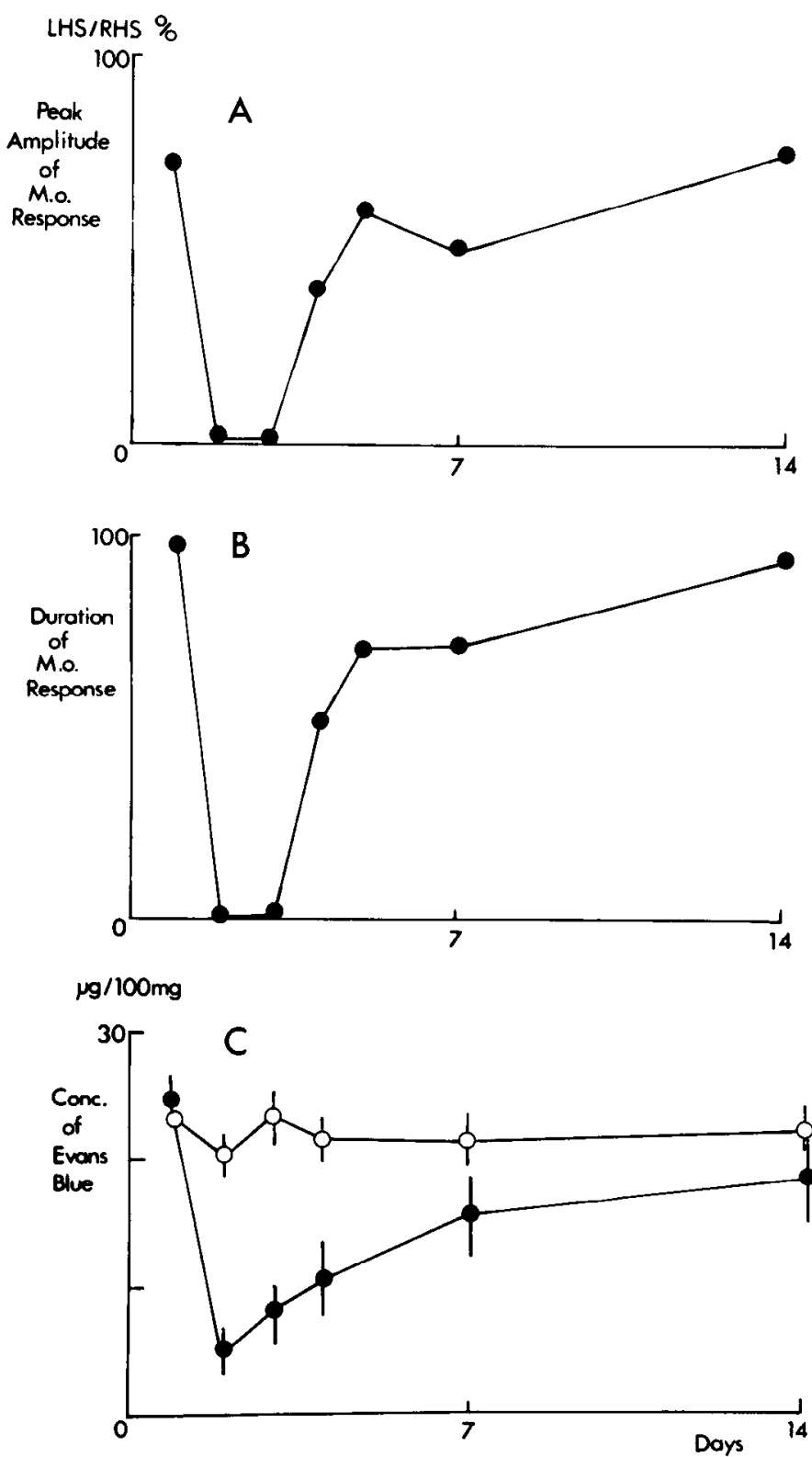

Figure 7. Time course of depression of reflex withdrawal and the neurogenic edema response to mustard oil painted on the skin of the foot following $10^{-4} \mathrm{M}$ vinblastine to the sciatic nerve. $A$ and $B$ show the pcak amplitude and duration, respectively, of the reflex EMG response recorded from the hamstring muscles expressed as a percentage of the LHS (treated) value over the RHS (untreated) value. $C$ shows the concentration of Evans blue in $\mu \mathrm{g} / 100 \mathrm{mg}$ wet weight of skin $15 \mathrm{~min}$ after mustard oil application. The LHS (treated) values are shown as solid circles and the RHS (untreated) values are shown as open circles. Note how the three time courses, from 1 to 14 days after treatment, parallel one another.

example of total abolition of the late response to turpentine, seen in 4 of 10 cases on day 2 to 4 . On day 7 , the response was totally normal $(n=3)$.

Neurogenic edema. The absence of chemically induced flexor reflexes was almost exactly paralleled by a blockade of neurogenic edema (Fig. $7 C$ ). The appearance of a blue patch of skin due to plasma extravasation of Evans blue could not be obtained either by application of mus- tard oil $(n=17)$ or by antidromic stimulation of the treated sciatic nerve at $\mathrm{C}$ fiber strength $(n=2)$ in $10^{-4}$ $M$ vinblastine-treated rats. The onset of the block was the same as changes in the flexor reflex beginning on day 2 following treatment, with a clear recovery by day 7 (Fig. 7C). Three of six rats showed some neurogenic edema on day 4 to 5 . The two phenomena, chemical sensitivity and neurogenic edema, were not always totally linked in individual animals. During this recovery phase it was possible to see an EMG response to mustard oil but with no neurogenic edema or, indeed, vice versa. Nevertheless, the time courses were very similar. At the lowest doses $\left(10^{-5} \mathrm{M}\right)$ reflex responses to mustard oil and neurogenic edema were completely normal ( $n=4$, day $3)$.

\section{Discussion}

The results raise a number of interesting points concerning the role of axonal transport in sensory nerves and the use of the transport blocker, vinblastine.

The combination of electron microscopy and chemical staining showed that the only dose that effectively blocked axonal transport in both $\mathrm{A}$ and $\mathrm{C}$ fibers while not producing degeneration was $10^{-4} \mathrm{M}$ vinblastine, and threfore the results at this dose contribute most to the questions we set out to investigate. However, before discussing this, it is worth examining the effects of higher doses of vinblastine. A $5 \times 10^{-4} \mathrm{M}$ dose produces considerable degeneration of both large and small fibers in the nerve, as was reflected in the failure of conduction, sensory loss, and signs of paralysis in some rats. Dziegielewska et al. (1976) have previously found that $10^{-4} \mathrm{M}$ vinblastine results in paralysis and conduction failure 20 hr after injection into rat sciatic nerves. Curiously, though, Csillik et al. (1978) found that $10^{-3} \mathrm{M}$ had no effect on conduction or on the morphology of the nerve. This variability in the sensitivity to vinblastine could perhaps be due to the degree of dissection of the nerve and its sheath (Fink et al., 1973) or to strain differences, but degeneration was certainly very clear in the present experiments at this dose. Below $5 \times 10^{-4} \mathrm{M}$, we found that degeneration was largely restricted to $\mathrm{C}$ fibers, A fibers remaining more or less intact showing only a small compound action potential reduction. Whether the degeneration is a result of axonal transport blockade or due to a direct neurotoxic action of vinblastine is not known. The problem of degeneration following colchicine treatment has been a subject of much controversy for several years (Jackson and Diamond, 1977), but vinblastine has been used less often in studies of axonal transport. It is reported to produce fewer nonspecific effects than colchicine, and it is much more potent (Hanson and Edström, 1978). Nevertheless, there are many clinical reports of peripheral neuropathy following systemic vinblastine (Gottscholk et al., 1968; McLeod and Penny, 1969). It has been noted that $C$ fibers require much lower concentrations of colchicine than do A fibers to block axonal transport (Hanson and Edström, 1978), and this may relate to their greater sensitivity to degenerative changes.

It is noteworthy that, despite the fact that there is still considerable ultrastructural damage to $\mathrm{C}$ fibers with 2.5 
$\times 10^{-4} \mathrm{M}$, the latencies to withdrawal from hot water are not nearly as prolonged as those found with the highest dose $\left(10^{-4} \mathrm{M}\right)$. This perhaps reflects the importance of small myelinated $\mathrm{A} \delta$ fibers in the transmission of noxious thermal information (Burgess and Perl, 1973) or that a small $\mathrm{C}$ afferent volley can still produce a large central response. Neonatal capsaicin treatment, which destroys up to $90 \%$ of all primary afferent $\mathrm{C}$ fibers, also produces a surprisingly small deficit of noxious thermal responsiveness (see Fitzgerald, 1983a).

More interesting is the fact that, at the low dose of $10^{-4} \mathrm{M}$, when axon transport is blocked, there is no effect at all on the thermal or mechanical sensitivity of the skin in that region tested by foot withdrawal latency or EMG responses. This suggests that thermal and mechanically sensitive nociceptors do not require the continuous arrival of "trophic" material from the cell body in order to function. It should be stressed that $10^{-4} \mathrm{M}$ vinblastine blocked transport in both $\mathrm{A}$ and $\mathrm{C}$ fibers. This is known because AChE on enzyme limited to A fibers as well as FRAP limited to $C$ fibers had their transport blocked. In striking contrast to this, two other properties of peripheral receptors apparently do fail on blockade of axon transport, chemical sensitivity, and the ability to produce neurogenic edema. Neurogenic edema is an inflammatory reaction to certain chemicals that requires the functional integrity of $\mathrm{C}$ fibers in the skin (Jancso et al., 1967). It can be mimicked by antidromic stimulation of $\mathrm{C}$ fibers in sensory nerves (Jancso et al., 1967). An active substance, transported from the cell body, is thought to be released from $\mathrm{C}$ fiber terminals via an axon reflex to produce this edema (Lewis, 1942; Chapman et al., 1961; Jancso, 1968; Morton and Chahl, 1980). SP (Lembeck and Gamse, 1982) and ATP (Holton, 1959) are two possible candidates. Here we have shown directly that neurogenic edema is greatly reduced following blockade of axon transport. Parallel to this block in neurogenic edema was a block of chemical sensitivity, which is also a property of $\mathrm{C}$ fiber terminals (Handwerker, 1976). The EMG reflex responses to mustard oil, a specific C fiber irritant (Jancso, 1968), were absent, and turpentine, possibly a more general nerve terminal irritant, also produced a reduced response. The time course of this depression matched that of the blockade of neurogenic edema. These two properties of $\mathrm{C}$ fibers have previously been linked (Jancso, 1968). Following neonatal or adult systemic treatment with capsaicin, neurogenic edema and chemical sensitivity always disappear, whereas depression of thermal and mechanical noxious responses is variable (see Fitzgerald, 1983a). More recently, the onset of neurogenic edema and the onset of chemical sensitivity have been shown to develop in parallel, 10 days after birth, which is much later than mechanical and thermal pain sensitivity (Fitzgerald, 1983b).

The present results, together with these previous studies, suggest that the chemical sensitivity of $\mathrm{C}$ fibers and the capacity to generate neurogenic edema are functions of $\mathrm{C}$ fibers that differ from their role as simple carriers of noxious mechanical and thermal information. Neurogenic edema could be considered as a "trophic" function of $\mathrm{C}$ fibers, assisting in the reaction of the skin to injury, whereas chemical sensitivity is both the afferent arc of this axon reflex and a way in which the CNS is informed about the chemical environment of the C-terminals. It is possible that, in addition to electrical activity generated by tissue injury, there are afferent chemical signals that operate over a longer time course (Wall, 1983).

The blockade of these $\mathrm{C}$ fiber functions takes at least $24 \mathrm{hr}$ to occur, presumably because of remaining stores of material in the distal portion of the nerve. Since the remaining length is approximately $50 \mathrm{~mm}$, this material must be traveling at $2 \mathrm{~mm} / \mathrm{hr}$ at maximum, a figure which agrees well with the average transport of substance $P$ in guinea pig sciatic nerve (Brimijoin et al., 1980). Recovery of function occurred in 4 to 5 days, at the same time as recovery of axon transport. Axon transport blockade of FRAP by application of colchicine to the sciatic nerve has also been shown to recover in 4 to 7 days (Krikorian et al., 1980).

The extent to which the change in conduction in $\mathrm{C}$ fibers contributes to the changes in chemical sensitivity found at the low dose $\left(10^{-4} \mathrm{M}\right)$ is not clear. The $C$ wave is undoubtedly depressed following this dose although, as stated earlier, the $\mathrm{C}$ fibers are structurally intact. Furthermore, the wave is still small, although recovering, 14 days later, when axon transport and $\mathrm{C}$ fiber terminal function have apparently fully recovered. The $C$ wave is difficult to interpret. It is highly dependent on synchronization and consists of a mixture of action potentials and afterpotentials (Douglas and Ritchie, 1962). Only single unit recording is really sufficient to give information on whether $\mathrm{C}$ fibers are conducting, but this method suffers greatly from sampling problems.

Apart from events distal to the axonal transport block, our results also show that local peripheral nerve vinblastine treatment can trigger retrograde changes in the cell bodies and the central terminals of the sensory neuron. The depletion of FRAP from $C$ fiber terminals in the dorsal horn confirms the results of Csillik and KnyiharCsillik (1982). The effects on SP were less clear-cut, but in most cases there was no depletion. FRAP and SP are both contained in the small B-type dorsal root ganglion cells and transported both peripherally and centrally. Each is contained in a separate, nonoverlapping population, however, so that it is quite possible that FRAPcontaining neurons are a particularly sensitive group (Nagy and Hunt, 1982). Reduced synthesis and depletion of chemicals from the neuron presumably occurs because of a changed signal from the periphery. This might be the appearance of a new exogenous signal (Kristensson and Olsen, 1974), the reversal of normally orthogradely transported material at the site of treatment (Bisby and Bulger, 1977), or the blockade of normally retrogradely transported material such as nerve growth factor (Stoeckel et al., 1975).

The depletion of FRAP in the dorsal horn by vinblastine is not accompanied by any transmission failure in the $\mathrm{C}$ fiber terminals. We have shown this by recording the input to dorsal horn cells from electrical $\mathrm{A}$ and $\mathrm{C}$ fiber stimulation and from mechanical and thermal stimulation of the skin following vinblastine treatment. This does not support the thesis of Csillik and Knyihar-Csillik (1982) that the dropout of FRAP is accompanied by the 
TABLE I

A summary of the physiological, chemical, and anatomical consequences of different manipulations of the sciatic nerve of the rat: cut, crush, local capsaicin treatment $(49 \mathrm{mM})$, and local vinblastine treatment $\left(10^{-4} \mathrm{M}\right)$

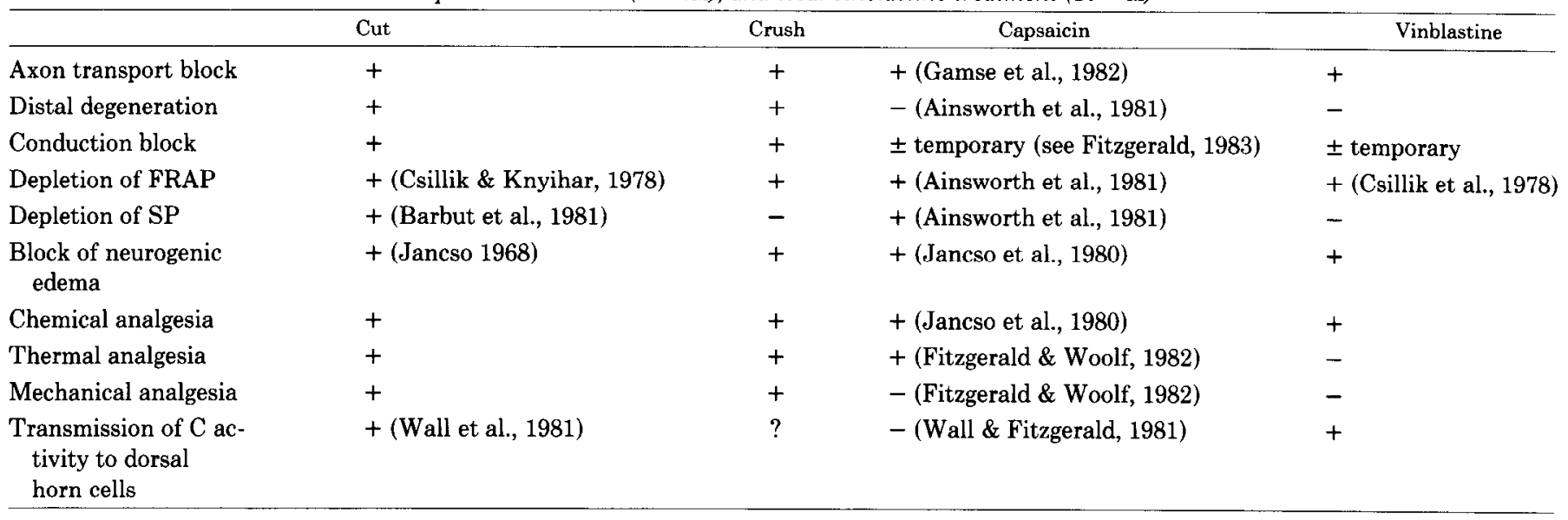

uncoupling of the first synapse in the nociceptive pathway. Sectioning a nerve which also results in FRAP depletion has also been shown not to reduce its ability to excite second-order cells on electrical stimulation (Wall et al., 1981).

It is interesting at this point to compare the effects of cutting a peripheral nerve, crushing it, and treating it locally with capsaicin or with vinblastine. This comparison is summarized in Table I. All four treatments produce axon transport block (Gamse et al., 1982) and depletion of FRAP (Csillik and Knyihar, 1978; Ainsworth et al., 1981), but only cutting and capsaicin treatment produce reliable SP depletion (Ainsworth et al., 1981; Barbut et al., 1981). In terms of chemical changes, vinblastine could be seen as a "temporary" crush. Capsaicin, like vinblastine, does not produce lasting $\mathrm{C}$ fiber conduction blockade (Wall and Fitzgerald, 1981) and also results in reduction of neurogenic edema and chemical sensitivity (Jancso et al., 1980). Unlike vinblastine, however, it results in transmission failure of $\mathrm{C}$ fiber terminals in the dorsal horn and long-lasting thermal analgesia (Fitzgerald and Woolf, 1982). It therefore appears that capsaicin has other actions in addition to axon transport blockade.

Much useful information could be gained by measuring the axoplasmic transport quantitatively, using radioactive tracers. In the present study only qualitative measures were used, but in the future it would be clearly important to correlate electrophysiological and morphological characteristics with the exact status of axon transport. Nevertheless, two clear conclusions can be drawn from this study. The first is that chemical sensitivity and the production of neurogenic edema by $\mathrm{C}$ fiber terminals depends on continuous transport of materials to the axon terminals. It would be interesting to establish whether these properties are a "trophic" action of $\mathrm{C}$ fibers on the skin, monitoring tissue injury and assisting in its repair. The role of $\mathrm{C}$ fiber sensory innervation of the skin may be analogous to the maintenance of taste buds by the lingual nerve (Sloan et al., 1983). The second conclusion is that peripheral axon transport blockade can produce some, but not all, of the retrograde changes in sensory neurons that follow peripheral nerve injury.
Despite depletion of FRAP, no functional defects were detected in the central nerve terminals. This confirms earlier findings that continuous retrograde transport of materials from the periphery is not necessary for normal central transmission (Wall et al., 1981). It would be interesting to test the suggestion that such transport is important in the more complex regulation of connectivity and receptive field organization among second-order neurons in the dorsal horn (Wall, 1983), but that would require a longer-lasting blockade of transport.

\section{References}

Ainsworth, A., P. Hall, P. D. Wall, G. Allt, L. M. Mackenzie, S. Gibson, and J. Polak (1981) Effects of capsaicin applied locally to adult peripheral nerve. II. Anatomy and enzyme and peptide chemistry of peripheral nerve and spinal cord. Pain 11: 379-388.

Barbut, D., J. M. Polak, and P. D. Wall (1981) Substance P in the spinal cord dorsal horn decreases following peripheral nerve injury. Brain Res. 205: 289-298.

Bisby, M. A., and V. T. Bulger (1977) Reversal of axonal transport at a nerve crush. J. Neurochem. 29: 313-320.

Brimijoin, S., J. M. Lundberg, E. Brodin, T. Hökfelt, and G. Nilsson (1980) Axonal transport of substance $P$ in the vagus and sciatic nerves of the guinea-pig. Brain Res. 191: 443457.

Burgess, R., and E. R. Perl (1973) Cutaneous mechanoreceptors and nociceptors. In Handbook of Sensory Physiology, A. Iggo, ed., Vol. 1, pp. 29-78, Springer-Verlag, Berlin.

Chapman, L. F., A. O. Ramos, B. S. Goodell, and H. G. Wolff (1961) Neurohumoral features of afferent fibres in man. Arch. Neurol. 4: 617-650.

Csillik, E., and E. Knyihar (1978) Biodynamic plasticity in the Rolando substance. Prog. Neurobiol. 10: 203-230.

Csillik, B., and E. Knyihar-Csillik (1982) Reversibility of microtubule inhibitor-induced transganglionic degenerative atrophy of central terminals of primary nociceptive neurons. Neuroscience 7: 1149-1154.

Csillik, B., E. Knyihar, I. Jojart, A. A. Elshiekh, and I. Por (1978) Perineural microtubule inhibitors induce degenerative atrophy of central nociceptive terminals in the Rolando substance. Res. Commun. Chem. Pathol. Pharmacol. 21: 467484.

Cull, R. E. (1975) Role of axonal transport in maintaining central synaptic connections. Exp. Brain Res. 24: 97-101. 
Devor, M., and D. Claman (1980) Mapping and plasticity of acid phosphatase afferents in rat dorsal horn. Brain Res 190 : 17-28.

Douglas, W. W., and J. M. Ritchie (1962) Mammalian nonmyelinated nerve fibres. Physiol. Rev. 42: 297-334.

Dziegielewska, K. M., N. R. Saunders, C. A. N. Evans, P. O. Skacel, C. J. Häggendal, P. O. Heiwall, and A. B. Dahlström (1976) Effects of colchicine and vinblastine on axonal tran port of choline acetyltransferase in rat sciatic nerve. Acta Physiol. Scand. 96: 486-494.

Fink, B. R., M. R. Byers, and M. E. Middaugh (1973) Dynamics of colchicine effects on rapid axonal transport and axonal morphology. Brain Res. 56: 299-311.

Fitzgerald, M. (1982) The contralateral input to the dorsal horn of the spinal cord in the decerebrate spinal rat. Brain Res. 236: 275-287.

Fitzgerald, M. (1983a) Capsaicin and sensory neurons-A review. Pain 15: 109-130.

Fitzgerald, M. (1983b) The development of C-fibre function in neonatal rats. J. Physiol. (Lond.) 341: 58P.

Fitzgerald, M., and C. J. Woolf (1982) The time course and specificity of the changes in the behavioral and dorsal horn cell responses to noxious stimuli following peripheral nerve capsaicin treatment in the rat. Neuroscience 7: 2051-2056.

Gamse, R., U. Petsche, F. Lembeck, and G. Jancso (1982) Capsaicin applied to peripheral nerve inhibits axoplasmic transport of substance $\mathrm{P}$ and somatostatin. Brain Res. 239: 447-462.

Gottscholk, P. G., P. J. Dyk, and J. M. Kiely (1968) Vinca alkaloid neuropathy: Nerve biopsy studies in rats and in man. Neurology 18: 875-882.

Handwerker, H. O. (1976) Influences of algogenic substances and prostaglandins on the discharges of unmyelinated cutaneous nerve fibres identified as nociceptors. Adv. Pain Res. Ther. 1: 41-46.

Hanson, M., and A. Edström (1978) Mitosis inhibitors and axonal transport. Int. Rev. Cytol. Suppl. 7: 373-402.

Holton, P. (1959) The liberation of adenosine triphosphate of antidromic stimulation of sensory nerves. J. Physiol. (Lond.) 145: 494-504.

Jackson, P., and J. Diamond (1977) Colchicine block of cholinesterase transport in rabbit sensory nerves without interference with the longterm viability of the axons. Brain Res. 130: $579-584$

Jancso, G., E. Kiraly, and A. Jancso-Gabor (1980) Direct evidence for an axonal site of action of capsaicin. Naunyn Schmiedebergs Arch. Pharmacol. 313: 91-94.

Jancso, N. (1968) Desensitization with capsaicin and related acylamides as a tool for studying the function of pain receptors. In Pharmacology of Pain, R. K. S. Lim, ed., pp. 33-55, Pergamon Press, Oxford.
Jancso, N., A. Jancso-Gabor, and J. Szolcsanyi (1967) Direct evidence for neurogenic inflammation and its prevention by denervation and by pretreatment with capsaicin. Br. J. Pharmacol. Chemother. 31: 138-151.

Kiernan, J. A. (1981) Histological and Histochemical Methods: Theory and Practice, Pergamon Press, Oxford.

Krikorian, J. G., L. Guth, and C. P. Barrett (1980) Transport of acid phosphatase in normal and transected rat sciatic nerve. Exp. Neurol. 70: 665-674.

Kristensson, K., and Y. Olsen (1974) Retrograde transport of HRP in transected axons. I. Time relationships between transport and induction of chromatolysis. Brain Res. 79: 101109 .

Lembeck, F., and R. Gamse (1982) Substance $P$ in peripheral sensory processes. Ciba Found. Symp. 91: 35-54.

Lewis, T. (1942) Pain, Macmillan, New York.

Lieberman, A. R. (1971) The axon reaction: A review of the principal features of perikaryal responses to axon injury. Int. Rev. Neurobiol. 14: 49-124.

McLeod, J. G., and R. Penny (1969) Vinblastine neuropathy: An electrophysiological and histological study. J. Neurol. Neurosurg. Psychiatry 32: 297-304.

Morton, C. R., and L. A. Chahl (1980) Indirect evidence for the importance of axonal transport in maintenance of stores of the mediator of neurogenic oedema. Naunyn Schmiedebergs Arch. Pharmacol. 314: 277-280.

Nagy, J. I., and S. P. Hunt (1982) Fluoride-resistant acid phosphatase-containing neurones in dorsal root ganglia are separate from those containing substance $P$ or somatostatin. Neuroscience 7: 89-97.

Schwartz, J. H. (1979) Axonal transport: Components, mechanisms, and specificity. Annu. Rev. Neurosci. 2: 467-504.

Sloan, H. E., S. E. Hughes, and B. Oakley (1983) Chronic impairment of axonal transport eliminates taste responses and taste buds. J. Neurosci. 3: 117-123.

Stoeckel, K., M. Schwab, and H. Thoenen (1975) Specificity of retrograde transport of nerve growth factor in sensory neurons. A biochemical and morphological study. Brain Res. 89: $1-14$.

Thomas, P. K., and R. H. M. King (1974) The degeneration of unmyelinated axons following nerve section: An ultrastructural study. J. Neurocytol. 3: 497-512.

Wall, P. D. (1983) The Textbook of Pain, Churchill Livingstone, London.

Wall, P. D., and M. Fitzgerald (1981) Effects of capsaicin applied locally to adult peripheral nerve. I. Physiology of peripheral nerve and spinal cord. Pain 11: 363-377.

Wall, P. D., M. Fitzgerald, and S. J. Gibson (1981) The response of rat spinal cord cells to unmyelinated afferents after peripheral nerve section and after changes in substance $P$ levels. Neuroscience 6: 2205-2215. 